\title{
Calculation of the probability of quantum transitions in the system by the method of functional integration
}

\author{
Alexander Biryukov ${ }^{1, *}$, Igor Matveev ${ }^{2}$, and Mark Shleenkov ${ }^{1, * *}$ \\ ${ }^{1}$ Samara National Research University, 34, Moskovskoye shosse, Samara, 443086, Russia \\ ${ }^{2}$ Povolzhskiy State University of Telecommunications and Informatics, 23, L. Tolstoy str., Samara, \\ 443010, Russia
}

\begin{abstract}
The probabilities of quantum transitions are described by integrals over trajectories in the space of energy states of a system exposed to an electromagnetic field. We describe quantum transitions in hydrogen atom.
\end{abstract}

\section{Introduction}

Describing the interaction between a quantum system and an intense electromagnetic field is quite difficult when using means of the theory of perturbation [1,2]. Developing nonperturbative ways of describing the evolution of a quantum system exposed to an elec- tromagnetic field is therefore important. One non-perturbative approach is the formalism of functional integration (integrating over trajectories) [3, 4].

In this work, the probability of transitions over any interval of time between the states of a quantum system interacting with an electromagnetic field are presented in the form of an integral over trajectories in the space of the energy states of a system. Since it is difficult to obtain an analytical solution for these integrals, we propose an algorithm for their numerical integration that is based on recurrent relationships.

\section{Probabilities of transitions in the formalism of functional integrals in the space of energy states}

Let us consider a multilevel quantum system that interacts with an electromagnetic field. It is described by Hamiltonian $\hat{H}$, written as

$$
\hat{H}=\hat{H}_{s y s t}+\hat{V}
$$

where $\hat{H}_{\text {syst }}$ is the Hamiltonian of the quantem system that determines its stationary states $|n\rangle$ with energies $E_{n}$ :

$$
\hat{H}_{s y s t}|n\rangle=E_{n}|n\rangle, \quad\left\langle n^{\prime} \mid n\right\rangle=\delta_{n^{\prime} n} \quad \sum_{n=1}^{N}|n\rangle\langle n|=1, \quad n=1,2, \ldots
$$

\footnotetext{
*e-mail: biryukov_1@mail.ru

**e-mail: shleenkov@list.ru
} 
where $\hat{V}$ is the operator of interaction between the system and the electromagnetic field, and $N$ is the number of quantum states of the system. We shall describe a system at moment $t$ by state vector $|t\rangle$, whose equation of evolution in our concept of interaction has the form

$$
\begin{array}{r}
|t\rangle=\hat{U}_{D}|0\rangle, \quad \hat{U}_{D}(t)=\hat{T} \exp \left[-\frac{l}{\hbar} \int_{0}^{t} \hat{V}_{D}(\tau) d \tau\right] \\
\hat{V}_{D}(\tau)=\exp \left[\frac{l}{\hbar} \hat{H}_{\text {syst }} \tau\right] \hat{V}_{D}(\tau) \exp \left[-\frac{l}{\hbar} \hat{H}_{\text {syst }} \tau\right]
\end{array}
$$

where $\hat{U}_{D}(t), \hat{V}_{D}(\tau)$ are the operators of evolution and interaction in Dirac's concept.

We shall assume that in our model, the operator of interaction between an electron of the system and a plane electromagnetic wave is determined by the expression

$$
\hat{V}(\tau)=e \hat{x} E_{x}^{0}(\tau) \cos (\Omega \tau),
$$

where $\hat{x}, e$ are the coordinate and the electron charge, respectively; $E_{x}^{0}(\tau)$ is the projection of the wave amplitude onto axis $x$, wich can change over time; and $\Omega$ is the field's frequency of vibration. In the basis presented by vectors (2), we write operator (4) in the form

$$
\hat{V}_{D}=\sum_{n^{\prime}, n=1}^{N} \frac{1}{2} \Omega_{n^{\prime} n}^{R}(\tau)\left(\exp \left[-l\left(\Omega-\omega_{n^{\prime} n}\right) \tau\right]+\exp \left[\imath\left(\Omega+\omega_{n^{\prime} n}\right) \tau\right]\right)\left|n^{\prime}\right\rangle\langle n|,
$$

where $\Omega_{n^{\prime} n}^{R}(\tau)=e\left|\left\langle n^{\prime}|\hat{x}| n\right\rangle\right| E_{x}^{0}(\tau) / \hbar$ is the Rabi frequency and $\omega_{n^{\prime} n}=\left(E_{n^{\prime}}-E_{n}\right) / \hbar$ is the frequency of a transition between levels.

Our problem is to determine probabilities $P\left(n_{f}, t \mid n_{i n}, 0\right)$ of transitioning between quantum states $\left|n_{i n}\right\rangle$ and $\left|n_{f}\right\rangle$ when $t=0$ and $t>0$ for a system under the influence of an electromagnetic field.

We write Eq. (3) in the energy representation, using the basis presenter by vectors (2),

$$
\left\langle n_{f} \mid t\right\rangle=\sum_{n_{i n}=1}^{N}\left\langle n_{f}\left|\hat{U}_{D}(t)\right| n_{i n}\right\rangle\left\langle n_{i n} \mid 0\right\rangle
$$

where $\left\langle n_{f}\left|\hat{U}_{D}(t)\right| n_{\text {in }}\right\rangle$ is the amplitude of a quantum transition from state $\left|n_{i n}\right\rangle$ at moment $t=0$ to state $\left|n_{f}\right\rangle$ at moment $t>0$. Probability $P\left(n_{f}, t \mid n_{i n}, 0\right)$ of this quantum transition is correspondingly expressed as

$$
P\left(n_{f}, t \mid n_{i n}, 0\right)=\left\langle n_{f}\left|\hat{U}_{D}(t)\right| n_{i n}\right\rangle\left\langle n_{\text {in }}\left|\hat{U}_{D}^{\dagger}(t)\right| n_{f}\right\rangle .
$$

To calculate probability of transition (8), we must specify the explicit form of transition amplitude $\left\langle n_{f}\left|\hat{U}_{D}(t)\right| n_{\text {in }}\right\rangle$ for finite interval of time $t$, using the group properties of operator $\hat{U}_{D}(t)$ and the entirety of state vectors $|n\rangle$, which we write in the form

$$
\left\langle n_{f}\left|\hat{U}_{D}(t)\right| n_{i n}\right\rangle=\sum_{n_{1}, \ldots, n_{K-1}}^{N} \prod_{k=1}^{K}\left\langle n_{k}\left|\hat{U}_{D}(t)\right| n_{k-1}\right\rangle,
$$

where $t_{k}=t, n_{K}=n_{f}, t_{0}=0, n_{i n}=n_{0}$ and $\left|n_{k}\right\rangle$ are the vectors of state at moment $t_{k}$;

$$
\left\langle n_{k}\left|\hat{U}_{D}(t)\right| n_{k-1}\right\rangle=\left\langle n_{k}\left|\hat{T} \exp \left[-\frac{l}{\hbar} \int_{0}^{t} \hat{V}_{D}(\tau) d \tau\right]\right| n_{k-1}\right\rangle, \quad t_{k}>t_{k-1}
$$


is the amplitude of the quantum transition between states $\left|n_{k-1}\right\rangle$ and $\left|n_{k}\right\rangle$ for the brief interval of time $t_{k}-t_{k-1}$. Once amplitude $\left\langle n_{k}\left|\hat{U}_{D}(t)\right| n_{k-1}\right\rangle$ of transition is plotted in explicit form according to formula (9), we find explicit amplitude of transition for any range of times $t$.

We now show for proposed model of interaction (6) that amplitude (10) of quantum transition for the fairly brief interval of time $t_{k}-t_{k-1}=\Delta t_{k}$ has the form [5]

$$
\left\langle n_{k}\left|\hat{U}_{D}\left(t_{k}, t_{k-1}\right)\right| n_{k-1}\right\rangle=\int_{0}^{1} \exp \left[-\imath S\left[n_{k}, t_{k} ; n_{k-1}, t_{k-1} ; \xi_{k-1}\right]\right] d \xi_{k-1},
$$

where operation $S\left[n_{k}, t_{k} ; n_{k-1}, t_{k-1} ; \xi_{k-1}\right]$ in units of $\hbar$ is defined by the expression

$$
\begin{array}{r}
S\left[n_{k}, t_{k} ; n_{k-1}, t_{k-1} ; \xi_{k-1}\right]= \\
2 \pi\left(n_{k}-n_{k-1}\right) \xi_{k-1}+\Omega_{n_{k} n_{k-1}}^{R} \Delta t_{k}\left(\cos \left[2 \pi\left(n_{k}-n_{k-1}\right) \xi_{k-1}-\left(\Omega-\omega_{n_{k}, n_{k-1}} \bar{t}_{k}\right)\right]+\right. \\
\left.+\cos \left[2 \pi\left(n_{k}-n_{k-1}\right) \xi_{k-1}-\left(\Omega+\omega_{n_{k}, n_{k-1}} \bar{t}_{k}\right)\right]\right) .
\end{array}
$$

where $\bar{t}_{k}=\frac{1}{2}\left(t_{k}+t_{k-1}\right)$ and $\omega_{n_{k} n_{k-1}}=\frac{1}{\hbar}\left(E_{n_{k}}-E_{n_{k-1}}\right)$ is frequency of thatsition between levels.

The amplitude of a system transitioning between quantum states for any interval of time $\mathrm{t}$ is determined by (9) with allowance for formulas (11) and (12):

$$
\left\langle n_{f}\left|\hat{U}_{D}\right| n_{i n}\right\rangle=\hat{R}_{K} \exp \left[-l S\left[n_{f}, n_{K-1}, \xi_{K-1} ; \ldots ; n_{1}, n_{i} n, \xi_{0}\right]\right],
$$

where

$$
S\left[n_{f}, n_{K-1}, \xi_{K-1} ; \ldots ; n_{1}, n_{i} n, \xi_{0}\right]=\sum_{k=1}^{K} S\left[n_{k}, t_{k}, n_{k-1}, t_{k-1}, \xi_{k-1}\right]
$$

$\hat{R}_{K}=\prod_{k=0}^{K} \hat{R}_{k}$ and operators $\hat{R}_{k}$ have the form

$$
\begin{array}{r}
\hat{R}_{k}=\sum_{n_{k}=1}^{N} \sum_{m_{k}=1}^{N} \int_{0}^{1} d \xi_{k} \int_{0}^{1} d \chi_{k}, \quad k=1,2, \ldots \\
\hat{R}_{0}=\int_{0}^{1} d \xi_{0} \int_{0}^{1} d \chi_{0},
\end{array}
$$

It is clear that the conjugate amplitude of transition has exactly the same structure with a change in the sign before imaginary unit i. Substituting formula (13) and conjugate amplitude into expression (8), we obtain the probability of a quantum transition from state $\left|n_{i n}\right\rangle$ in to state $\left|n_{f}\right\rangle$ for finite interval of time $t$.

\section{Calculation of transitions probabilities for hydrogen atom}

The proposed formalism is applicable to the description of quantum transitions in a hydrogen atom under the action of a monochromatic polarized electromagnetic wave field. The probabilities of transition between quantum states $2 \mathrm{~s}, 3 \mathrm{~s}, 3 \mathrm{p}, 4 \mathrm{~s}, 4 \mathrm{p}, 4 \mathrm{~d}$ with energies respectively $E_{2}, E_{3}, E_{4}$ for the time interval $\mathrm{t}$ will be calculated by the formula (8). The probability amplitudes corresponding to these transitions will be determined by (11) where the action $S$ is concretized by the model. 
For the numerical calculation of the amplitude, we present it in the form of a recurrent formula where $S$ is defined by (12). We calculate the probabilities of hydrogen atom staying at the levels $3 \mathrm{~s}, 3 \mathrm{p}, 4 \mathrm{~s}, 4 \mathrm{p}, 4 \mathrm{~d}$, initially prepared in the state $2 \mathrm{~s}$. The electic field $E$ is directed along the $z$ axis. Non-zero Rabi frequencies between quantum states for $E=0.5 \cdot 10^{6} \mathrm{~V} / \mathrm{m}$ are $\Omega_{2 s, 3 p}^{R} / 2 \pi=1.80 \mathrm{GHz}, \Omega_{2 s, 4 p}^{R} / 2 \pi=0.75 \mathrm{GHz}, \Omega_{3 s, 4 p}^{R} / 2 \pi=3.22 \mathrm{GHz}, \Omega_{3 p, 4 s}^{R} / 2 \pi=$ $2.87 \mathrm{GHz}, \Omega_{3 p, 4 d}^{R} / 2 \pi=3.98 \mathrm{GHz}$.
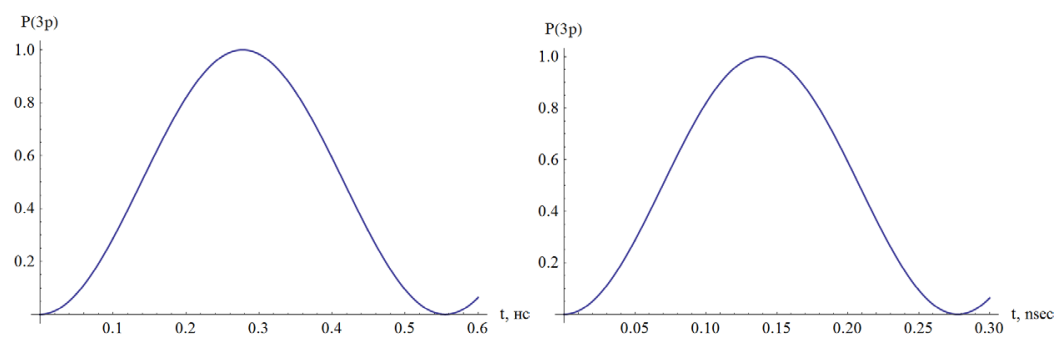

Figure 1. The probability of detecting an atom in a state of 3p. $E=0.5 \cdot 10^{6} \mathrm{~V} / \mathrm{m}$ (left), $10^{6} \mathrm{~V} / \mathrm{m}$ (right)
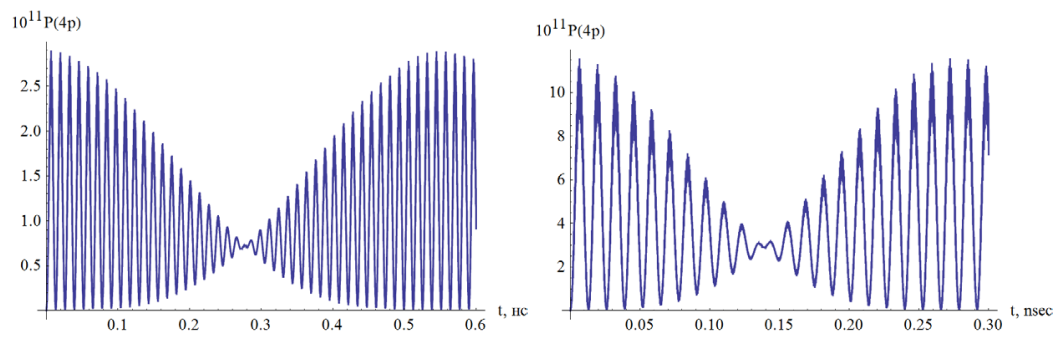

Figure 2. The probability of detecting an atom in a state of 4 p. $E=0.5 \cdot 10^{6} \mathrm{~V} / \mathrm{m}$ (left), $10^{6} \mathrm{~V} / \mathrm{m}$ (right)
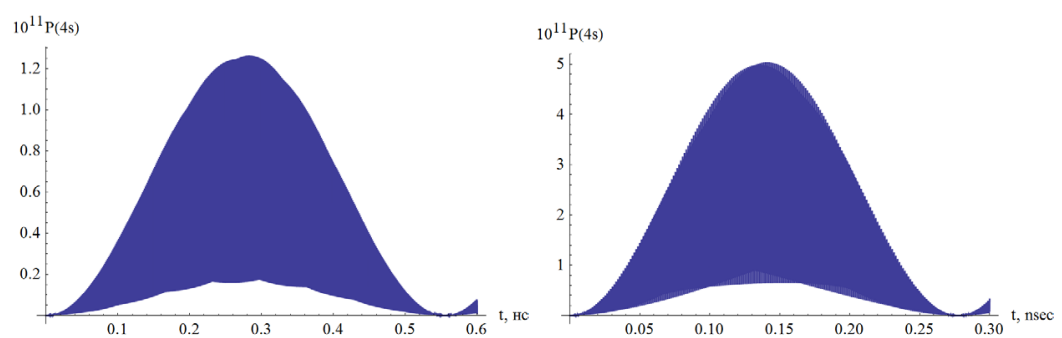

Figure 3. The probability of detecting an atom in a state of $4 \mathrm{~s} . E=0.5 \cdot 10^{6} \mathrm{~V} / \mathrm{m}$ (left), $10^{6} \mathrm{~V} / \mathrm{m}$ (right)
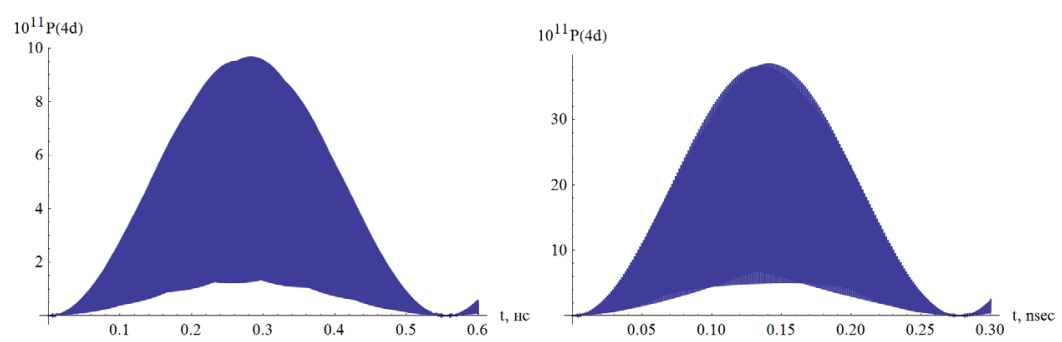

Figure 4. The probability of detecting an atom in a state of $4 \mathrm{~d} . E=0.5 \cdot 10^{6} \mathrm{~V} / \mathrm{m}$ (left), $10^{6} \mathrm{~V} / \mathrm{m}$ (right) 
The results of the numerical calculation are presented in figures 1-4 (there are the results for the amplitude of the electric field $E=0.5 \cdot 10^{6} \mathrm{~V} / \mathrm{m}$ (left) and $E=10^{6} \mathrm{~V} / \mathrm{m}$ (right).

The figure 1 illustrate the classical Rabi oscillations. The figure 2 shows the probability of detection of the system in the $4 p$ state. Here we illustrate the process of multiphoton transition from the $2 \mathrm{~s}$ state. Figures 3 and 4 shows the probability of system detection in the $4 \mathrm{~s}$ and $4 \mathrm{~d}$ states from the $3 \mathrm{p}$ state.

Thus, the proposed the method successfully describes both single-photon and multiphoton transitions and transitions through virtual levels. The presence of multiphoton processes is confirmed by the probability of transitions increasing with an increase in the electric field strength. The presence of multiphoton processes is confirmed by the dependence of the probability of transitions on the electric field.

\section{Conclusion}

The proposed method successfully describes quantum transitions in the system under the action of an electromagnetic wave. This formalism describes both single-photon processes and multi-photon processes. The analysis shows that it is suitable for describing a wide class of quantum systems.

\section{References}

[1] Ge, W., Hemmer, P.R., Suhail Zubairy, M., Phys. Rev. A, 87, 023818 (2013)

[2] Biryukov, A.A., Danilyuk, B.V., Proc. SPIE, 7024, 702405 (208)

[3] Feynman, R.P. and Hibbs, A.R. Quantum Mechanics and Path Integrals (McGraw-Hill, 1965) 365

[4] Biryukov, A.A., Shleenkov, M.A., Vestn. Samar. Gos. Tekh. Univ., Ser. Fiz.-Mat. Nauki, 19, 221 (2015)

[5] Biryukov, A. A., Degtyareva Ya. V., Shleenkov M. A., Bulletin of the Russian Academy of Sciences: Physics, 82, 1565-1569 (2018) 